\section{Las aguas del centenario}

La infraestructura de la ciudad tiene múltiples aristas, unas más aparentes que otras. Hoy la movilidad y las comunicaciones son el centro de la discusión, tal como la relación entre el agua (lluvia, servida, potable) y la ciudad lo fue en el pasado. Emprendidas hace un siglo, la revisión de esas obras de infraestructura hídrica revela insospechados alcances: iniciativas funcionales que probablemente sin proponérselo ban alimentado y cautelado ciertas cualidades de nuestro paisaje urbano.

Palabras clave: Urbanismo, Larraín Bravo, alcantarillado, acequias, agua, Santiago, parques, canalización.

The city's infrastructure has many angles, some more obvious than others. Mobility and communication are as central to today's debate as the city's relationship to water (rain, drinking water and sewage), was in the past. A review of public water works carried out a century ago reveals som unexpected impacts among the functional initiatives that, probably unintentionally, have nourished and protected some qualities of our urban landscape.

Key words: Town planning, Larrain Bravo, sewage systems, drainage, water, Santiago, parks, canal systems.
Infraestructura y desarrollo urbano en Santiago de Chile a comienzos del siglo $X^{1}$

Es un hecho frecuentemente subrayado que la ciudad de Santiago está marcada -desde sus iniciospor una estructura vial y predial a la vez que por un sistema de acequias y canales, que servirán tanto para efectos de regadío, como para la evacuación de las aguas servidas. En una zona semiárida, esta vasta y densa red, desarrollada en los siglos sucesivos, posibilitó su riego y su paulatina transformación en una interminable sucesión de huertos ${ }^{2}$. Así, no sólo el paisaje que la circunda, sino la propia ciudad, experimentó la acción transformadora del agua a lo largo de su historia.

La ciudad del centenario: infraestructura y embellecimiento urbano

A comienzos del siglo XX, la celebración del centenario de la independencia impulsará una serie de reformas y obras urbanas, muchas de las cuales venían perfilándose desde fines del siglo XIX. Durante la segunda mitad de ese siglo, el país se había empeñado en la construcción de una capital republicana. Un siglo de independencia debía poner de manifiesto una ciudad que exhibiera los atributos de una auténtica capital (Pérez, Rosas, 2002).

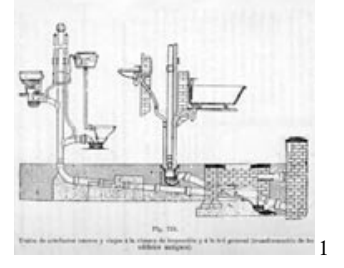

Versión revisada de la ponencia presentada por los autores en The
$11^{\text {h }}$ International Planning History Conference 2004 / Planning models and the culture of cities. Barcelona, julio de 2004

Al destacar la dimensión de embellecimiento de las transformaciones urbanas ocurridas alrededor de 1910, se ha descuidado la conexión que muchas de ellas tienen con operaciones de infraestructura, y que el objetivo de lograr una ciudad higiénica fue, probablemente, uno de sus más potentes impulsores. Las nuevas infraestructuras preparan el terreno para el desarrollo metropolitano y posibilitan transformaciones radicales en los usos de la ciudad. La localización de corredores para los servicios técnicos resultan ser elementos clave de la organización urbana y condicionan sus procesos de crecimiento y desarrollo inmobiliario. La complejidad y riqueza de este proceso adquiere especial relevancia en uno de sus aspectos clave: aquél que tiene relación con las infraestructuras de conducción y utilización de las aguas. Los cambios que producirá en la ciudad la canalización del río, a fines del siglo XIX, y la construcción de un moderno sistema de evacuación de aguas servidas y aguas lluvia, son decisivos para entender las mutaciones de la ciudad del centenario.

\section{Vicuña Mackenna y Ansart: el río y las} cloacas

La reforma urbana ideada a fines del siglo XIX por el intendente Benjamín Vicuña Mackenna
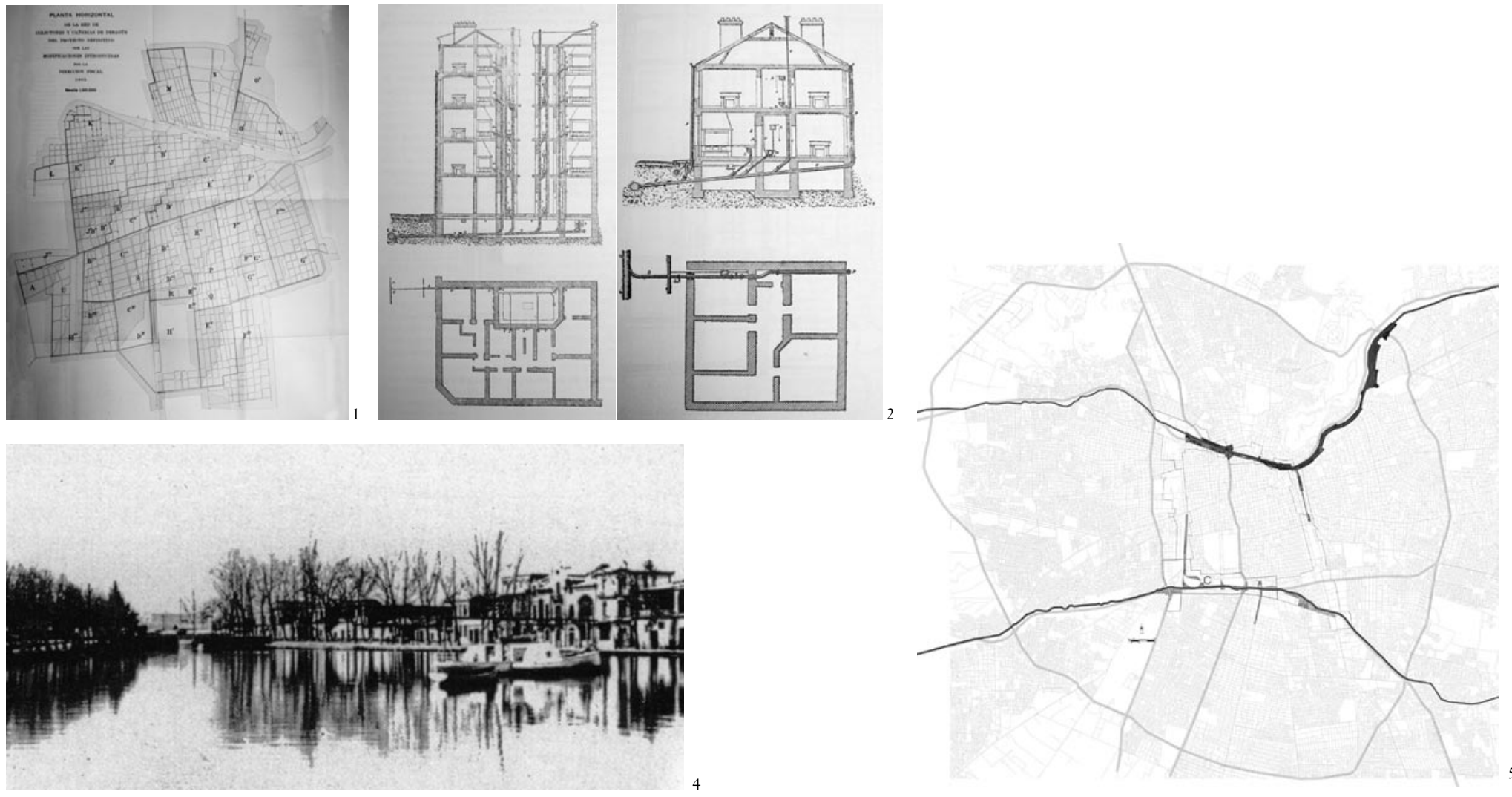


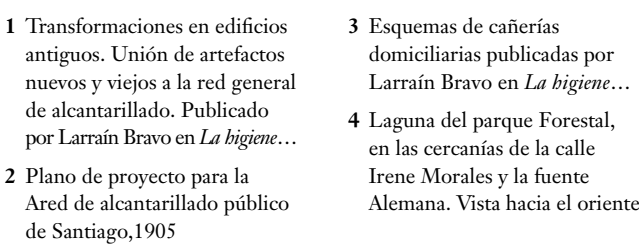

5 Cumbrera y limahoya del valle

de Santiago. El río Mapocho y

el zanjón de La Aguada.

Proyecto parque Bicentenario

La Aguada, 2003
Estas circunstancias han sido specialmente destacadas, entre otros, ${ }_{3}^{\text {por Ricardo Astaburuag }}$ de Castro, M.A.; "Las ideas de ciudad tras las intervenciones sobre el río" en Pérez, Fernando; Arquitectura y cultura en el Santiago de Ansart. Seminario de investigación, EAU constituye el antecedente más directo de las obras del centenario. El carácter visionario y la ambición de su propuesta le impidieron concretarla cabalmente, aunque muchas de sus ideas continuaron alimentando los proyectos urbanos impulsados en las siguientes décadas. En este contexto, la participación del ingeniero francés Ernesto Ansart no ha sido suficientemente destacada. Sus ideas y su capacidad técnica le permitieron sentar las bases de algunos proyectos clave de Vicuña Mackenna, fuertemente ligados a problemas de infraestructura. Es el caso de las obras viales e hidráulicas para la transformación del cerro Santa Lucía y las diversas propuestas para la canalización del río.

La reforma urbana propuesta por Vicuña Mackenna queda registrada en el texto $L a$ transformación de Santiago publicado en 1872, que expone 20 propuestas de intervención urbana. Para cada una de ellas, el intendente constituyó comités de trabajo integrados por técnicos, autoridades y vecinos influyentes, involucrando, con notable visión política, a los actores fundamentales. De gran actualidad, la argumentación utilizada por Vicuña Mackenna justifica las propuestas única y exclusivamente en términos prácticos, y recurriendo frecuentemente a cálculos económicos. Incluso intervenciones literalmente de embellecimiento urbano, como la construcción de plazas y parques o la plantación de árboles, vienen justificadas en función de obtener una ciudad más higiénica y una rentabilidad económica en tal operación. Un número muy importante de los proyectos incluidos en La transformación de Santiago son obras de infraestructura, y varios se refieren al tratamiento urbano de las aguas. De hecho, el primero es la canalización del río Mapocho y otros dos refieren a la reconstrucción -O modernización- del sistema de alcantarillado: la canalización de la acequia de San Miguel y la construcción de la cloaca máxima de Negrete. En adición a estos proyectos, se propone un ensanche de la red de agua potable.

Ansart había dedicado una energía considerable al proyecto de canalización del Mapocho, sometido desde la colonia a la construcción de tajamares como protección de las inundaciones. De hecho, se conocen dos proyectos de su autoría relacionados con este problema. En el primero, proponía una canalización que incluía un desnivel al interior de la caja del río, incluyendo una nueva calle, inundable en el caso de crecidas. En el segundo, proponía desviar el curso del río hacia el norte generando un nuevo paseo en el antiguo curso, produciendo una nueva Alameda en el borde norte del centro de la ciudad ${ }^{3}$. Refiriéndose al proyecto de Ansart para la canalización del Mapocho, Vicuña Mackenna subraya que los terrenos liberados por la canalización permitirían realizar un brillante negocio al municipio. Como es sabido, las obras de canalización se llevaron a cabo más tarde con un modelo mucho más pragmático. Después de dos alternativas estudiadas por el ingeniero Valentín Martínez, las obras se concretan en 1888, ganando terrenos al norte y el sur del nuevo curso canalizado. El destino de los terrenos subidos de nivel a través de la tierra obtenida de las excavaciones, incluyendo la construcción del parque Forestal, será fundamental para el desarrollo de la ciudad en los años siguientes.

Ricardo Larraín Bravo: la higiene, la ciudad y el alcantarillado del centenario

Las convicciones higienistas del siglo XIX irrumpen en el siglo $\mathrm{XX}$, adquiriendo un mayor alcance y una formulación más sistemática. Este hecho queda bien ejemplificado en el surgimiento de una figura como la de Ricardo Larraín Bravo. Su cátedra de Higiene en las construcciones habla por sí misma del rango que el tópico llega a adquirir en materias urbanas. Probablemente sea su actividad pedagógica la que motive a Ricardo Larraín a escribir su monumental obra La bigiene aplicada a las construcciones (en adelante La bigiene...) publicada precisamente en los años del centenario (1909-10).

Estructurada en tres tomos y más de dos mil páginas, La bigiene... constituye un intento monumental por integrar en torno a este concepto las ideas arquitectónicas y urbanas vigentes en el período y expresa la convicción que determinados aspectos técnicos tienen la capacidad de dar forma a la arquitectura y transformar la ciudad. En una compleja combinación de catastro e informe técnico, texto educativo, recopilación de casos y manual práctico, permite aquilatar cuánto la idea de una ciudad higiénica y saludable fue determinante en las transformaciones urbanas del centenario. Las teorías higienistas adquieren así una nueva dimensión operativa que se manifiesta en las infraestructuras de agua potable y alcantarillado, y una dimensión teórica no menos significativa. Estas se manifiestan en el debate acerca del tópico y en el estudio y evaluación de las propuestas que finalmente se realizan.

El texto registra la magnitud de la operación y el largo trámite que exigió de instalar un sistema de alcantarillado moderno en Santiago. Entre las muchas propuestas que se habían sucedido, sin llegar a concretarse, destacan el proyecto de la Dirección General de Obras Públicas de 1890, el proyecto del ingeniero Valentín Martínez de 1893, el proyecto del ingeniero Gaspar Roufosse de 1899 y el proyecto de los ingenieros Chiesa y Pinchón de 1900. Ese mismo año, el gobierno designa una comisión para estudiar diversas alternativas para el alcantarillado de Santiago. Dicha comisión consideró necesaria la elaboración de un nuevo proyecto, utilizando el caudal del río Mapocho para alimentar los colectores y facilitar su lavado. En conformidad con estas bases, el ingeniero Santa María, miembro de la comisión, entregó en noviembre de 1901 el Proyecto definitivo del alcantarillado de Santiago. Este permitía la presentación de nuevos proyectos o la utilización de los existentes, incorporando en las bases los planos elaborados por Martínez, Roufosse, Chiesa y Pinchón y, naturalmente, el del ingeniero Santa María.

El estudio técnico y financiero de las tres propuestas fue encargado por el Gobierno a una comisión de cuatro miembros incluyendo a Ricardo Larraín Bravo. La gravitación de éste fue determinante en la selección, modificaciones y recomendaciones de la propuesta, aconsejando aceptar la propuesta B de la empresa Batignolles - Fould. En el tomo segundo de La bigiene... Larraín Bravo reafirma la significación de la trama de la ciudad y el sentido de la pendiente general como determinantes en el diseño de la nueva red. En efecto, Santiago presentaba condiciones favorables para el trazado del alcantarillado por su estructura urbana de damero y por la existencia de un caudal continuo y suficiente de agua.

La articulación eficiente de la cuadricula fundacional, orientada según los puntos cardinales, con la diagonal sur poniente en la que se alcanzaba la pendiente máxima, dará como resultado la estructura en zig-zag que caracteriza la versión final de la red. Se localizan así, de preferencia, los nuevos colectores por las calles norte-sur, de mayor pendiente, y los conductos de menor importancia por las calles oriente poniente. Larraín Bravo se había opuesto tenazmente a aprovechar las antiguas acequias de la ciudad -que corrían desde la Colonia por la medianería oriente-poniente de las manzanaspara el trazado del alcantarillado, aduciendo las múltiples dificultades que el interior de las manzanas presentaba al trazado de la red, apartándose en ello de las recomendaciones de carácter pragmático del propio Senado de la República, en el sentido de aprovechar las antiguas acequias. Propone, en cambio, que las 
${ }^{4}$ Ver al respecto Echevería, C.; "De la naturaleza como escenario geográfico Dubois y el parque Forestal", resultad del seminario de investigación en Pérez, Fernando; Constructores y viajero presencia de extranjeros en construcción de la ciudad en Chile 1840
Este proyecto ha sido desarrollado y Estudios Urbanos de la Universida Católica de Chile entre 2002 y 2004, en una iniciativa liderada por Pablo Allard Rodrigo Pérez de Arce, José Rosas Bonifacio Fernández, junto al equip SEREX coordinado por Anarés Camus de DECON PU.C. nuevas obras se practiquen en la vía pública, de propiedad común, privilegiando la disposición en zig-zag que aprovecha mejor la pendiente del valle.

La ciudad y las aguas: el alcantarillado y el parque

Las consecuencias del trazado del nuevo alcantarillado son múltiples y van mucho más allá de la disponibilidad de un sistema de evacuación de desechos y aguas lluvia que funciona eficientemente hasta hoy mismo. Entre ellas, el refuerzo de la trama de damero y el cambio en la estructura de frentes de la manzana tradicional. La construcción del nuevo alcantarillado consagró el trazado vial colonial, e hizo muy difícil la introducción de trazados alternativos -como aquellos que incluían diagonales- particularmente apreciados en los años del centenario. La primacía del trazado en zig-zag dejó en igualdad de condiciones los cuatro frentes de manzana: todos ellos pudieron acceder de manera equivalente a los servicios de alcantarillado. La nueva situación difería radicalmente de las condiciones previas, en que el acceso a las acequias que corrían por el centro de la manzana, favorecía a las propiedades situadas en sentido norte - sur por sobre aquellas oriente - poniente.

Es importante también fijar la atención en el punto de origen del sistema de alcantarillado, en el extremo oriente de la ciudad. El punto de contacto de la red de alcantarillado con el río -donde se introducen los flujos de lavado del sistema- se sitúa, de manera natural, en el arranque de la cañada. Allí surgiría la plaza Italia, punto de articulación entre el sector oriente y el sector central tradicional de Santiago. Ella no sólo será un punto significativo de la operación urbana del centenario, sino que anunciará el futuro desarrollo de la ciudad. En efecto, la plaza Italia representa el extremo oriente de un conjunto de monumentos situados en la nueva ribera sur ganada al río Mapocho, transformada en el parque Forestal. Entre ellos, se cuentan la nueva Estación Mapocho, el Museo de Bellas Artes, el monumento donado por Francia, la fuente donada por Alemania, el monumento italiano y la nueva Estación Pirque. La lista hace pensar en un conjunto vinculado al hermoseamiento de la ciudad Sin embargo, ambas estaciones de ferrocarril tienen un carácter claramente infraestructural Además, la posibilidad de crear un parque longitudinal está directamente vinculada a la canalización del Mapocho. La plantación del parque Forestal, y la operación inmobiliaria en los terrenos aledaños, concreta, casi literalmente, las ideas que Vicuña Mackena había enunciado treinta años antes: la canalización del río sería, a la vez, una operación de higienización y un negocio inmobiliario. Además de surgir de una operación de canalización, el parque Forestal fue, en sí mismo, configurado a partir del agua. El paisajista Dubois se encontró con una difícil tarea cuando debió diseñar, a partir de 1901, un parque sobre un basural y antiguo curso de río, parcialmente ocupado por ranchos. Frente a tales circunstancias, tuvo la idea -bastante genial- de inundar porciones significativas del parque aprovechando la proximidad del río, creando un paisaje notable con pocos medios y en un tiempo mínimo ${ }^{4}$.

Este nuevo borde urbano, sobre el cual se localizan las principales operaciones arquitectónicas del centenario, anticipa la construcción de la avenida Costanera y el desarrollo de la ciudad hacia el oriente. El surgimiento del parque Forestal, uno de los hechos urbanos más destacables de la ciudad, pone de relieve una vez más el discurso higienista desde el cual dichas operaciones fueron justificadas, y la íntima vinculación entre un proceso de embellecimiento urbano y obras de infraestructura.

\section{Infraestructura y ciudad: de nuevo las aguas} La íntima relación de obras de infraestructura con el desarrollo y la forma de la ciudad no es novedad de este siglo. Recordar el modo en que operaciones como la canalización del río Mapocho y la construcción del alcantarillado de Santiago tuvieron consecuencias que van mucho más allá del objetivo técnico que las animaba, es particularmente significativo cuando se aproxima la celebración del bicentenario y se agitan las aguas de la polémica en torno al río y la infraestructura vial. La presencia del parque Forestal como uno de los episodios más notables de Santiago, junto al barrio que lo rodea, ponen de relieve que infraestructura y calidad urbana, entendida en el sentido más amplio, no son concepciones necesariamente contrapuestas. $\mathrm{Al}$ aproximarnos a la celebración del bicentenario, la presencia de grandes proyectos de infraestructura, aunque de diferente escala y naturaleza, vuelven a plantear la discusión sobre las oportunidades que ellos representan para el desarrollo urbano. En los albores del bicentenario, la ciudad vuelve a revisar sus fronteras internas $y$, curiosamente, vuelve a pensarse en parques inundables. Así ocurre con el proyecto parque Bicentenario La Aguada ${ }^{5}$
(2003) en el contexto del Plan Maestro Anillo Metropolitano de Santiago. Situado en el corredor vacante dejado por el antiguo ferrocarril de circunvalación, y vinculado al zanjón de $\mathrm{La}$ Aguada, otro protagonista destacado del sistema hidráulico de la ciudad, el proyecto propone, una vez más, el aprovechamiento de un curso de agua y sus riberas como generador de un espacio público y desarrollo urbano. Una versión contemporánea de las ideas de Vicuña Mackenna y Ansart. ARQ

Bibliografía: Astaburuaga, Ricardo; "El agua canalizada", "La casa huerto" y "La cuadrícula". En Morfología de Chile y sus ciudades. Ril Editores, Santiago, 2002, pp. 54, 57 y 94. / Larraín Bravo, Ricardo; La bigiene aplicada a las construcciones: alcantarillado, agua potable, saneamiento, calefacción, ventilación. Ed. Cervantes, Santiago, 1909. / Pérez Oyarzun, Fernando y Rosas, José; "Cities within the City: urban and architectural transfers in Santiago, Chile”. En Almandoz, Arturo, (Editor); Planning Latin American capital cities 1850-1950. Routledge, Londres, 2002, pp. 107-138. / Vicuña Mackenna, Benjamín; La transformación de Santiago. Imprenta de la Librería de El Mercurio, de Orestes L. Tornero, Santiago, 1872. 